\title{
Bounded Rationality: Static versus Dynamic
}

\author{
Approach
}

Suren Basov ${ }^{1}$

December 6, 2002

${ }^{1}$ The University of Melbourne, Melbourne, Victoria 3010, Australia. JEL classification: C0, D7. 


\begin{abstract}
Two kinds of theories of the boundedly rational behavior are possible. Static theories focus on stationary behavior and do not include any explicit mechanism for temporal change. Dynamic theories, on the other hand, explicitly model the fine-grain adjustments made by the subjects in response to their recent experiences. The main contribution of this paper is to argue that the restrictions usually imposed on the distribution of choices in the static approach are generically not supported by an explicit adjustment mechanism.
\end{abstract}




\section{INTRODUCTION}

Past decades have witnessed growing empirical evidence that calls into question the utility maximization paradigm. For a description of systematic errors made by experimental subjects, see Arkes and Hammond (1986), Hogarth (1980), Kahneman, Slovic, and Tversky (1982), and Nisbett and Ross (1980), and the survey papers by Payne, Bettman, and by Pitz and Sachs (1984). On the basis of this and similar evidence, Conlisk (1996) forcefully argued for the incorporation of bounded rationality in economic models. For an early work that tried to model boundedly rational decision making in a variety of economic contexts see, for example, Alchian (1950), Simon (1957), and Nelson and Winter (1982).

One may take two approaches to modelling bounded rationality. In the static approach individuals choose better alternatives more frequently than the inferior ones. It was introduced in economics by Luce (1959). It is typical for this approach to impose intuitive restrictions on choice probabilities and study probability distributions that satisfy these restrictions. Such probabilistic choice models have already found their application in economics. See, for example, McKelvey, Palfrey (1995, 1998), Chen, Friedman, Thisse (1997), Anderson, Goeree, and Halt (1998), Offerman, Schram, Sonnemans (1998), 
and Anderson, Goeree, and Halt (2001).

In the dynamic approach individuals are assumed to adjust their choices over time in the directions that appear beneficial. The dynamic approach originated in the work of Arrow and Hurwicz (1960) and is represented, for example, by the papers of Foster and Young (1990), Fudenberg and Harris (1992), Kandori, Mailath, Rob (1993), Young (1993), Friedman and Yellin (1997), Anderson, Goeree, and Holt (1999), and Friedman (2000).

The dynamic approach is a more fundamental one. It tries to model the fine-grain adjustments made by the individuals on the basis of their current experiences. On a very general level, such adjustments produce a stochastic process on the choice set. The probability distribution of choices of the static approach can be naturally viewed as the steady state distribution of this stochastic process. For a study of a broad class of such processes, see Basov (2001).

An important question is whether the restrictions imposed on the probability distribution of choices by the static approach can be supported by a generic adjustment process of some class. A natural class of adjustment processes are locally improving processes. I call an adjustment process locally improving if the vector of expected change points into a direction of an 
increase of the utility. An adjustment process is payoff monotone if for any admissible choice set the density of the steady state distribution at point $x_{1}$ is greater than at point $x_{2}$ if and only if the payoff for alternative $x_{1}$ is greater then the payoff for alternative $x_{2}$. I show that although any payoff monotone rule is locally improving the reverse is not true. Moreover, assuming that the adjustment process is Markov, I show that the payoff monotonicity puts strong restrictions on its generator that have no a priori economic justification.

Another restriction that is often imposed on the steady state choices in static models is the Independence of Irrelevant Alternatives (IIA). I will show that in the dynamic context IIA leads to restrictions on the generator of the Markov process that are strictly weaker than those implied by the payoff monotonicity. That is any payoff monotone Markov process satisfies IIA. (Note, that requiring the payoff monotonicity and IIA to hold for the stationary distributions over any admissible choice set makes them the properties of the adjustment process rather then of the steady state). Though the restrictions for the generator of the Markov process implied by IIA are strictly weaker then those implied by the payoff monotonicity they are still strong enough to cast doubt on the IIA assumption. In fact, I prove that 
for a broad class of locally improving processes a generic process does not satisfy IIA. Moreover, a generic locally improving process that does satisfy IIA is not payoff monotone.

The above discussion implies that restrictions put on the probability density in the static approach are too strong. They are typically not supported by a dynamic adjustment approach. This undermines the static approach and calls for an explicit study of the adjustment rules.

This paper is organized as follows. Section 2 describes a broad class of stochastic adjustment processes. Section 3 studies that connection between the local improvement property, the payoff monotonicity, and the IIA. Section 4 concludes.

\section{A MODEL OF INDIVIDUAL BEHAVIOR}

Assume an individual repeatedly faces with a problem of choosing an alternative from a one-connected, compact set $\Omega \subset R^{n}$. I will call such sets admissible. She adjusts her choices gradually in response to her recent experiences. The adjustment rule produces a stochastic process on the choice space. The deterministic component of the process can be interpreted as an 
attempt to increase her payoff, while the random component can be interpreted as experimentation. I will assume that this process is Markov and that it possesses a generator. The last assumption is technical in nature and allows us to employ the continuous time technique.

To build a formal model of adjustment assume that $\Sigma$ be a sigma-algebra on $\Omega$ and for any $\Gamma \in \Sigma$, define $P(x(t), \Gamma, t, \tau)$ to be the transition probability, that is the probability that the individual who at time $t$ made a choice $x(t)$, will make a choice $w \in \Gamma$ at time $t+\tau$.

Assumption The following limit exists

$$
£=\lim _{\tau \rightarrow 0} \frac{P(x, \Gamma, \tau)-I}{\tau},
$$

where $I$ is the identity operator.

This assumption asserts that the Markov process has a generator, £, (Rogers and Williams, 1994) and allows us to use the continuous time technique. It can be shown (Kanan, 1979) that the generator has the form:

$$
£=\mu(x) \nabla+\Gamma(x) D^{2},
$$


where

$$
\begin{aligned}
\mu(x) & =\lim _{\tau \rightarrow 0} \frac{1}{\tau} E(x(t+\tau)-x(t)) \\
\Gamma(x) & =\lim _{\tau \rightarrow 0} \frac{1}{\tau} \operatorname{Var}(x(t+\tau)-x(t)) .
\end{aligned}
$$

Vector $\mu$ captures the deterministic trend of the adjustment rule, while matrix $\Gamma$ is the covariance matrix of the random experimentation. The Markov process is completely characterized by vector $\mu$ and matrix $\Gamma$. I will sometimes refer to it as process $(\mu, \Gamma)$. Assume that $\Sigma$ is the Borel sigma algebra. Then the evolution of the density of the choices is determined by the following system (Ito, 1992).

$$
\begin{gathered}
\frac{\partial f}{\partial t}+\operatorname{div}(\mu(x) f)=\frac{1}{2} \sum_{i, j=1}^{n} \frac{\partial^{2}\left(\Gamma_{i j}(x) f\right)}{\partial x_{i} \partial x_{j}} \\
\sum_{i=1}^{n}\left(\frac{1}{2} \sum_{j=1}^{n} \frac{\partial\left(\Gamma_{i j}(x) f\right)}{\partial x_{j}}-\mu_{i}(x) f\right) n_{i}(x)=0 \text { on } \partial \Omega .
\end{gathered}
$$

Here $n(x)$ is the unit vector normal to the boundary of the choice set $\partial \Omega$, (Ito, 1992). In the rest of the paper I will assume that matrix $\Gamma$ does not depend on $x$. The assumption is made for the sake of simplicity and does not seriously affect the results. The definition of $\Gamma$ implies that it is positively 
semi-definite. I will assume that it is positively definite. From an economic perspective it means that the experimentation has a full range.

Let us assume that the preferences of the individual are given by a twice continuously differentiable function $U(x)$ and that the set

$$
U_{C}=\{x \in \Omega: \nabla U(x)=0\}
$$

has no limit points.

Definition 1 A Markov adjustment process is called locally improving if

$$
\langle\mu(x), \nabla U(x)\rangle \geq 0 \text { for } \forall x \in \Omega \text {. }
$$

Here and throughout the paper $\langle\cdot, \cdot\rangle$ denotes the inner product of two vectors. In words, a process is locally improving if the vector of the expected adjustment of the choice points into a direction of an increase of the utility. It seems natural to require for the choice adjustment process to be locally improving. 
Let $f_{s, \Omega}(x)$ be the stationary solution of the system (5)-(6) normalized by

$$
\int_{\Omega} f_{s, \Omega}(x) d x=1
$$

when the choice space is $\Omega$. It is also known as the steady state distribution of the Markov process. It is well defined according to the following theorem (Ito, 1992).

Theorem 1 Assume matrix $\Gamma$ is positively definite. There exists unique twice continuously differentiable stationary normalized solution of system (5)-(6). Moreover, it is positive everywhere on $\Omega$ and asymptotically stable.

Theorem 1 allows us to make the following definition.

Definition 2 A Markov adjustment process is payoff monotone if for any choice set $\Omega \subset R^{n}$ and any $x_{1}, x_{2} \in \Omega$

$$
\left(f_{s, \Omega}\left(x_{1}\right) \geq f_{s, \Omega}\left(x_{2}\right)\right) \Leftrightarrow\left(U\left(x_{1}\right) \geq U\left(x_{2}\right)\right)
$$

In words, a Markov adjustment process is payoff monotone if for any sufficiently small $\varepsilon>0$ the steady state probability that the choice is in an 
$\varepsilon-$ ball centered at the point $x_{1}$ is higher then the probability that it is in an $\varepsilon-$ ball centered at point $x_{2}$ if and only if alternative $x_{1}$ is preferred to alternative $x_{2}$. Note that the payoff monotonicity refers to the process rather than to a particular steady state distribution because the latter depends on $\Omega$, while the payoff monotonicity requires (8) to hold for any $\Omega$.

Another important restriction often imposed in the static approach is IIA. Definition 3 A Markov adjustment process satisfies the IIA if for any two choice sets $\Omega_{1}$ and $\Omega_{2}$ and any $x \in \Omega_{1} \cap \Omega_{2}$

$$
f_{s, \Omega_{1}}(x)=f_{s, \Omega_{2}}(x)
$$

Note that Theorem 1 allows us to talk about IIA processes rather then the distributions satisfying IIA. In words, IIA states that the steady state probability density of a choice does not depend on what other choices are available. My next task is to investigate the connections between the payoff monotonicity, the IIA, and the property of the process of being locally improving. 


\section{LOCAL IMPROVEMENTS, THE PAYOFF MONOTONICITY, AND THE IIA}

In this Section I am going to investigate the connections between the payoff monotonicity, the IIA, and the property of the process of being locally improving. I am going to show that a generic payoff monotone process does not satisfy IIA and a generic payoff monotone process that does satisfy IIA is not payoff monotone. I am going to start with proving the following Lemma.

Lemma 1 A Markov process is payoff monotone if and only if for any $\Omega$ there exists a strictly increasing continuously differentiable function $g_{\Omega}: R \rightarrow$ $R_{+} /\{0\}$ such that

$$
f_{s, \Omega}(x)=g_{\Omega}(U(x))
$$

Proof. Consider a rational continuous preference order $\succeq$ defined by

$$
(x \succeq y) \Leftrightarrow(U(x) \geq U(y))
$$

The payoff monotonicity implies that $f_{s, \Omega}(x)$ is a utility function that represents the same preferences. Hence, there exists continuous strictly increasing 
function $g_{\Omega}: R \rightarrow R$ such that

$$
f_{s, \Omega}(x)=g_{\Omega}(U(x)) .
$$

According to Theorem $1, f_{s, \Omega}(\cdot)$ is positive on $\Omega$, hence $g_{\Omega}(\cdot)>0$. Let $\bar{U}$, $\bar{U}+\delta U \in \operatorname{Int}(U(\Omega))$ and $\delta U \neq 0$. Then $\exists x, x+\delta x \in \operatorname{Int}(\Omega)$ such that

$$
U(x)=\bar{U}, U(x+\delta x)=\bar{U}+\delta U
$$

Note that since set $U_{C}$ has no limit points it is always possible to find $x$ belonging to indifference map (13) such that $\nabla U(x) \neq 0$, which in turn allows to select $\delta x$ in such a way that $\langle\delta x, \nabla U(x)\rangle \neq 0$. Note that

$$
(\delta U \rightarrow 0) \Leftrightarrow(\delta x \rightarrow 0)
$$

Let $\delta x=a\|\delta x\|$, where $a$ is unit vector pointing in the direction $\delta x$. Then

$$
\lim _{\delta U \rightarrow 0} \frac{g_{\Omega}(\bar{U}+\delta U)-g_{\Omega}(\bar{U})}{\delta U}=\frac{\left\langle\nabla f_{s, \Omega}, a\right\rangle}{\langle\nabla U, a\rangle} .
$$

Equation (15) asserts that the limit on the left hand side exists, hence the 
function $g_{\Omega}(\cdot)$ is differentiable. Moreover, the derivative is continuous. The right hand side does not depend on $a$, which means that $\nabla f_{s, \Omega}$ is proportional to $\nabla U$ with the coefficient of proportionality $g_{\Omega}^{\prime}(U(x))$.

Q. E. D.

Next, I am going to characterize the payoff monotone adjustment processes. As we will see, the payoff monotonicity implies some connection between the deterministic part of the adjustment process, $\mu$, and its stochastic part, $\Gamma$. As a corollary, I will prove that any payoff monotone adjustment process is locally improving. The reverse, however, is not true. Moreover, I will describe a broad class of locally improving processes such that the measure of the payoff monotone processes within this class is zero. Hence, generically locally improving processes are not payoff monotone. To formalize these ideas let us start with proving the following Theorem.

Theorem 2 Consider a Markov process $(\mu, \Gamma)$ and assume that for $\forall x_{0} \in \Omega$ the following limit exists and is not equal to zero

$$
\lim _{x \rightarrow x_{0}} \frac{\langle\mu(x), \nabla U(x)\rangle}{\langle\nabla U(x), \Gamma \nabla U(x)\rangle} .
$$


The process is payoff monotone if and only if there exists a continuous function $c: R \rightarrow R_{+} /\{0\}$ such that

$$
\mu=c(U) \Gamma \nabla U
$$

Moreover, if the Hessian of $U$ has full rank $c(\cdot)$ is differentiable.

Roughly speaking (16) says that $\mu(x)=0$ at points where $\nabla U(x)=0$, that is there is no deterministic adjustment at the critical points of the utility function.

Proof. Suppose (17) holds. Define

$$
\xi(z)=2 \int_{0}^{z} c(y) d y .
$$

Then it is easy to check that

$$
f_{s, \Omega}(x)=\frac{\exp (\xi(U(x)))}{\int_{\Omega} \exp (\xi(U(y))) d y}
$$

is a normalized stationary solution of (5)-(6). According to Theorem 1 it is the unique normalized stationary solution. According to $(18), \xi^{\prime}(\cdot)=c(\cdot)>$ 
0. Hence,

$$
\left(f_{s, \Omega}\left(x_{1}\right) \geq f_{s, \Omega}\left(x_{2}\right)\right) \Leftrightarrow\left(U\left(x_{1}\right) \geq U\left(x_{2}\right)\right)
$$

so the adjustment process is payoff monotone.

Now, suppose that the adjustment process is payoff monotone. Then, according to Lemma 1, there exists a continuously differentiable strictly increasingfunction $g_{\Omega}: R \rightarrow R_{+} /\{0\}$ such that

$$
f_{s, \Omega}(x)=g_{\Omega}(U(x)) .
$$

Define vector

$$
\kappa=g_{\Omega}(U(x)) \mu(x)-\frac{g_{\Omega}^{\prime}(U(x))}{2} \Gamma \nabla U(x) .
$$

Then (5)-(6) implies that vector $\kappa$ satisfies

$$
\begin{aligned}
\text { div } \kappa & =0 \text { on } \Omega, \\
\langle\kappa, n\rangle & =0 \text { on } \partial \Omega .
\end{aligned}
$$

Moreover, definition of payoff monotonicity implies that (23)-(24) should hold 
for any $\Omega$. Hence, $\kappa=0$. Now (22) implies that

$$
\frac{g_{\Omega}^{\prime}(U(x))}{2 g_{\Omega}(U(x))}=\frac{\langle\mu(x), \nabla U(x)\rangle}{\langle\nabla U(x), \Gamma \nabla U(x)\rangle}
$$

provided that $\nabla U(x) \neq 0$. Taking into account (16) we can extend (24) on the entire of $\Omega$. The right hand side of (24) does not depend on $\Omega$, hence the left hand side should also not depend on $\Omega$. Introduce

$$
c(z)=\frac{g_{\Omega}^{\prime}(z)}{2 g_{\Omega}(z)}
$$

According to Lemma $1, c(\cdot) \geq 0$ and according to $(16)$ and $(24) c(\cdot) \neq 0$. Hence, $c(\cdot)>0$. Finally, putting $\kappa=0$ in $(22)$ and using the definition of $c(\cdot)$ we get

$$
\mu(x)=c(U(x)) \Gamma \nabla U(x) .
$$

Proof of differentiability of $c(\cdot)$ is similar to the proof of differentiability of $g_{\Omega}(\cdot)$ in Lemma 1 and is omitted.

Q.E.D.

An easy corollary of Theorem 2 is that any payoff monotone process is 
locally improving.

Corollary 1 If Markov process $(\mu, \Gamma)$ is payoff monotone and (16) holds it is locally improving.

Proof. According to Theorem 2, we can write

$$
\mu(x)=c(U(x)) \Gamma \nabla U(x) .
$$

for some positive real valued function $c(\cdot)$. Therefore,

$$
\langle\mu(x), \nabla U(x)\rangle=c(U(x))\langle\nabla U(x), \Gamma \nabla U(x)\rangle \geq 0 .
$$

Hence, the process is locally improving.

Q. E. D.

The reverse, however, is not true. Indeed, consider the following example.

Example 1. Assume that the choices made by the individual follow the stochastic process: 


$$
d x=\nabla U(x) d t+\Lambda d W
$$

when $x$ is an interior point of $\Omega$. Here $U(\cdot)$ is twice continuously differentiable function, which is interpreted as a utility function of the individual, $\Lambda$ is $n \times n$ matrix of full rank and $W=\left(W_{1}, \ldots, W_{n}\right)$ is a vector of independent standard Wiener processes. If $x \in B d(\Omega)$ the agent follows rule (30) whenever possible, and stays put otherwise. Note that process (30) is locally improving and $\mu$ and $\Gamma$ are given by

$$
\begin{gathered}
\mu(x)=\nabla U(x) \\
\Gamma=\Lambda^{T} \Lambda .
\end{gathered}
$$

The first term in (30) corresponds to a gradient dynamics and says that agents adjust their choices in the direction of the maximal increase of utility. The second term states that these choices are subject to random error or experimentation. This assumption implies that the errors are uncorrelated in time, though correlation among different components of $x$ is permitted and is given by the matrix $\Gamma=\Lambda^{T} \Lambda$. 
Let us assume $n=2$, and put

$$
\begin{aligned}
& U\left(x_{1}, x_{2}\right)=u_{1}\left(x_{1}\right)+u_{2}\left(x_{2}\right), \\
& \Lambda=\begin{array}{cc}
\sigma_{1} & 0 \\
0 & \sigma_{2}
\end{array} .
\end{aligned}
$$

Then

$$
f_{s, \Omega}(x)=C_{\Omega} \exp \left(\frac{u_{1}\left(x_{1}\right)}{\sigma_{1}^{2}}+\frac{u_{2}\left(x_{2}\right)}{\sigma_{2}^{2}}\right)
$$

Consider two choice vectors $x=\left(x_{1}, x_{2}\right)$ and $y=\left(y_{1}, y_{2}\right)$. Assume $u\left(x_{1}\right)=$ $u\left(x_{2}\right)=5, u\left(y_{1}\right)=4, u\left(y_{2}\right)=8, \sigma_{1}^{2}=1, \sigma_{2}^{2}=10$. Then $u(x)<u(y)$ but $f(x)>f(y)$.

It turns out that the situation illustrated by Example 1 is quite generic. To formalize this claim consider a class $P D$ of locally improving Markov adjustment processes $\left(\mu_{B}, \Gamma\right)$ where

$$
\mu_{B}=B \nabla U
$$

for some positive definite matrix $B$ with constant coefficients. (Matrix $B$ is called positive definite if for any $z \in R^{n} /\{0\}\langle z, B z\rangle>0$. Note that matrix 
$B$ is not required to be symmetric.) For fixed $\Gamma$ each such adjustment process can be considered to be an element of $R^{n^{2}}$. Endowing $R^{n^{2}}$ with Lebesque measure we can make the class $P D$ a measure space. I claim that under some weak assumption on function $U(\cdot)$ the payoff monotone adjustment processes have zero measure in this measure space, i. e. a generic locally improving process of class $P D$ is not payoff monotone.

Let $x_{0} \in \operatorname{Int} \Omega$ and

$$
I=\left\{x \in \Omega: U(x)=U\left(x_{0}\right)\right\}
$$

be the indifference surface passing through $x_{0}$.

Assumption (LI) There exist $n$ different points $x_{1}, . ., x_{n} \in I$ such that vectors $\nabla U\left(x_{1}\right), \ldots, \nabla U\left(x_{n}\right)$ are linearily independent.

Assumption LI basically says that the indifference surface has some curvature in all directions, namely the Hessian of the utility has a full rank. The following result holds. 
Lemma 2 Assume $n>1$ and Assumption LI holds. Then the Lebesque measure of the payoff monotone processes in the class $P D$ is zero.

Proof. According to Theorem 2, for each payoff monotone process in class $P D$ we can write

$$
B \nabla U(x)=c(U(x)) \Gamma \nabla U(x) .
$$

for some positive real valued function $c(\cdot)$. Fix $x_{0} \in \operatorname{Int} \Omega$ and let $x_{1}, . ., x_{n} \in I$ be such that $\nabla U\left(x_{1}\right), \ldots, \nabla U\left(x_{n}\right)$ are linearily independent. Assumption LI guarantees that such $x_{1}, . ., x_{n}$ exist. Let us introduce the following notation

$$
\begin{aligned}
U\left(x_{0}\right) & =U, U_{k j}=\frac{\partial U\left(x_{k}\right)}{\partial x_{j}} \\
y_{j}^{i} & =b_{i j}-c(U) \gamma_{i j},
\end{aligned}
$$

where $b_{i j}$ and $\gamma_{i j}$ are matrix elements of matrices $B$ and $\Gamma$ respectively. Then for a fixed $i$

$$
\sum_{j=1}^{n} U_{k j} y_{j}^{i}=0 .
$$

Since $\nabla U\left(x_{1}\right), \ldots, \nabla U\left(x_{n}\right)$ are linearily independent the unique solution of 
(36) is $y_{j}^{i}=0$. Since this is true for every $i,(35)$ implies

$$
B=c(U) \Gamma
$$

Since both $B$ and $\Gamma$ are constant matrices $c(U)=c>0$ is also constant. This means that the set of payoff monotone processes is a ray $B=c \Gamma$, which has measure zero in $P D$, provided $n>1$.

$$
\text { Q.E.D. }
$$

Recall that a Markov adjustment process satisfies the IIA property if the ratio of the probability that the choice is in an $\varepsilon-$ ball centered at the point $x_{1}$ to the probability that it is in an $\varepsilon-$ ball centered at point $x_{2}$ does not depend on whether some other choice $z$ is available, up to the order $o(\varepsilon)$. The following result holds.

Theorem 3 A Markov adjustment process satisfies IIA if and only if the Jacobi matrix of the vector field $\Gamma^{-1} \mu(x), D\left(\Gamma^{-1} \mu(x)\right)$, is symmetric for $\forall x \in$ $\operatorname{Int}(\Omega)$. 
Proof. Let us introduce vector $j$ by a formula:

$$
j(x)=-\nabla U(x) f(x)+\frac{1}{2} \Gamma \nabla f(x) .
$$

Then in the steady state, $j(x)$ should solve the following boundary problem

$$
\begin{gathered}
\operatorname{div}(j(x))=0 \\
\langle j(x), n(x)\rangle=0 \text { on } x \in \partial \Omega .
\end{gathered}
$$

The distribution $f$ is then determined by the system of first-order partial differential equations:

$$
j(x)=-\mu(x) f(x)+\frac{1}{2} \Gamma \nabla f(x) .
$$

The IIA property implies that a change in $\Omega$ will result in multiplication of $f$, and hence of $j$, by a constant, that is $j_{\text {new }}=C j_{\text {old }}$. This relation should hold at each point which belongs to the intersection of the new and the old choice space. Hence $j_{\text {new }}$ should solve the same boundary problem, but on a different domain. The only vector $j$ that would solve (39)-(40) for any domain is $j=0$. Hence IIA together with the definition of $j$ implies that 
$f(x, t)$ solves the system

$$
\mu(x) f(x)-\frac{1}{2} \Gamma \nabla f(x)=0 .
$$

or

$$
\frac{1}{2} \nabla \ln f(x)=\Gamma^{-1} \mu(x)
$$

The Jacobi matrix of the left hand side of (43) is the Hessian matrix of $\ln f(x)$. Since, according to Theorem1, $f(x)$ is positive and twice continuously differentiable this matrix is symmetric, so the Jacobi matrix of the right hand side should also be symmetric.

To prove the reverse, assume that the Jacobi matrix of $\Gamma^{-1} \mu(x)$ is symmetric and define $f(x)$ to be the solution of (43). According to the Frobenuous theorem in this case the solution exists and is unique up to multiplicative constant. It is easy to see that $f(\cdot)$ such defined solves (5)-(6). The constant is chosen from the normalization condition.

Q. E. D.

Theorem 3 shows that IIA requires some connection between the deterministic and stochastic part of Markov process to hold. This connection 
does not have any a priori economic justification and we should not expect it to hold in general. Moreover, as I will show shortly, IIA does not hold for a generic Markov processes from some broad class of the payoff monotone processes. To see this consider a class $P D S$ of Markov adjustment processes, which is obtained from $P D$ assuming that $B$ is symmetric. I can be naturally embedded in $R^{n(n+1) / 2}$. Let us endow this set with Lebesque measure. Then the following result holds.

Lemma 3 Assume $n>1$ and for any non-degenerate matrix $C$ there exist $i, k$ such that

$$
\frac{\partial^{2} U}{\partial x_{i}^{\prime} \partial x_{k}^{\prime}} \neq 0
$$

where

$$
x^{\prime}=C x .
$$

Then the Lebesque measure of the payoff monotone processes in the class PDS is zero.

Proof. Since both matrices $\Gamma^{-1}$ and $B$ are positive definite, there exists a non-degenerate matrix $C$ such that both $C^{T} \Gamma^{-1} C$ and $C^{T} B C$ are diagonal 
with all diagonal entries are strictly positive (Gantmakher, 1989). Let us denote the $i^{\text {th }}$ diagonal element of $\Gamma^{-1}$ as $1 / \sigma_{i}^{2}$ and the $i^{\text {th }}$ diagonal element of $B$ as $b_{i}$. Let $x^{\prime}=C x$. Then by Theorem 3 the process satisfies IIA if and only if

$$
\left(\frac{b_{i}}{\sigma_{i}^{2}}-\frac{b_{k}}{\sigma_{k}^{2}}\right) \frac{\partial^{2} U}{\partial x_{i}^{\prime} \partial x_{k}^{\prime}}=0
$$

Now (46) and (44) imply that there exists a pair of indices $i, k$ such that

$$
\frac{b_{i}}{\sigma_{i}^{2}}=\frac{b_{k}}{\sigma_{k}^{2}}
$$

This means that the dimension of the set of processes for which IIA holds is at least by one smaller then $n(n+1) / 2$ and therefore, such processes have Lebesque measure zero.

$$
\text { Q.E.D. }
$$

As one can see from the proof assumptions (44)-(45) can be weakened. Indeed, it is sufficient to require them to hold only for $C$ that brings $\Gamma^{-1}$ and $B$ to a diagonal form, rather than for any non-degenerate $C$. Economically, assumption (44) says that the utility is not additively separable in the components of vector $x$. If it is separable IIA will hold for any process in 
PDS. However, in that case the process will not result in the payoff monotone outcome for any $\Omega$ that contains a set $\left\{x \in R_{+}^{n}:\|x\|<\varepsilon\right\}$ for some $\varepsilon>0$ unless $\Gamma=\sigma^{2} I$. Note that this claim is stronger then the claim that the process is not payoff monotone. I will illustrate it for the case $n=2$. The generalization for higher dimensions is straightforward.

Example 2. This example generalizes Example 1. Let us assume that $\Gamma=\operatorname{diag}\left(\sigma_{1}^{2}, \sigma_{2}^{2}\right)$ with $\sigma_{1}^{2}<1<\sigma_{2}^{2}$ (this assumption is made for illustrative purposes and do not affect the results) and

$$
U\left(x_{1}, x_{2}\right)=u_{1}\left(x_{1}\right)+u_{2}\left(x_{2}\right) .
$$

where $u_{i}(\cdot)$ are twice continuously differentiable, increasing, unbounded from above functions satisfying $u_{i}(0)=0$. Then the stationary solution of system (5)-(6) is

$$
f_{s, \Omega}(x)=C \exp \left(\frac{u_{1}\left(x_{1}\right)}{\sigma_{1}^{2}}+\frac{u_{2}\left(x_{2}\right)}{\sigma_{2}^{2}}\right) .
$$

Select $u_{1}>0$ and $u_{2}>0$ in such a way that

$$
u_{1}+u_{2}=\frac{u_{1}}{\sigma_{1}^{2}}+\frac{u_{2}}{\sigma_{2}^{2}} .
$$


This is always possible under our assumption on $\sigma_{i}$ s. It is a matter of simple geometry to persuade oneself that the set of solutions of the system

$$
\left\{\begin{array}{l}
u_{1}^{\prime}+u_{2}^{\prime}>u_{1}+u_{2} \\
\frac{u_{1}^{\prime}}{\sigma_{1}^{2}}+\frac{u_{2}^{\prime}}{\sigma_{2}^{2}}>u_{1}+u_{2} \\
u_{1}^{\prime}>0, u_{2}^{\prime}>0
\end{array}\right.
$$

is nonempty. Let $\left(u_{1}^{*}, u_{2}^{*}\right)$ be such a solution. Our assumptions on functions $u_{i}(\cdot)$ guarantee that there exist $\left(x_{1}, x_{2}\right)$ and $\left(y_{1}, y_{2}\right)$ such that $u_{1}=u_{1}\left(x_{1}\right)$, $u_{2}=u_{2}\left(x_{2}\right), u_{1}^{*}=u_{1}\left(y_{1}\right)$, and $u_{2}^{*}=u_{2}\left(y_{2}\right)$. Moreover, for any $\varepsilon>0$ there exists $\delta>0$ such that if $u_{1}<\delta$ and $u_{2}<\delta$ then $\|x\|<\varepsilon$ and $\|y\|<\varepsilon$. Hence, $x, y \in \Omega$ and the steady state distribution is not payoff monotone.

This example easily generalizes to the case $n>2$. Indeed, let us assume that there exist $i$ and $j$ such that $\sigma_{i} \neq \sigma_{j}$ and the utility has a form

$$
U(x)=\sum_{k=1}^{n} u_{k}\left(x_{k}\right) .
$$

For $k \notin\{i, j\}$ fix $x_{k}$ at some admissible level and apply the above construction to components $i$ and $j$. 
Results obtained so far show that both the payoff monotonicity and IIA do not hold for a generic locally improving processes. However, there are in some sense more processes that satisfy IIA then that satisfy monotonicity. To make that sense precise we will start by proving the following theorem.

Theorem 4 Assume the Hessian of the utility function is non-degenerate.

Then any payoff monotone process satisfies IIA.

Proof. According to Theorem 2 payoff monotonicity implies that

$$
\mu(x)=c(U(x)) \Gamma \nabla U(x)
$$

for some differentiable function $c: R \rightarrow R_{+}$. But then the matrix element $D\left(\Gamma^{-1} \mu\right)_{i j}$ is given by

$$
\frac{\partial\left(\Gamma^{-1} \mu\right)_{i}}{\partial x_{j}}=c^{\prime}(U(x)) \frac{\partial U}{\partial x_{i}} \frac{\partial U}{\partial x_{j}}+c(U(x)) \frac{\partial^{2} U}{\partial x_{i} \partial x_{j}} .
$$

Hence, the matrix is symmetric and the process satisfies IIA.

Q.E.D. 
Consider the class $P D S$. Lemma 2 easily generalizes to this case, so the set of monotone processes within this set has dimension one. On the other hand, it is easy to see from the proof of Lemma 3 that the set of IIA processes has dimension at least $n(n+1) / 2-(n-1)$. Therefore for $n>1$, if we endow the space of all payoff monotone processes satisfying IIA by a Lebesque measure as a subset of $R^{k}$ for an appropriate $k$, the set of the payoff monotone processes will have measure zero measure in this space. To conclude we have shown that for a sufficiently broad class of locally improving processes a generic process does not satisfy IIA and a generic process that satisfies IIA is not payoff monotone. Note also that an IIA process need not be locally improving.

\section{DISCUSSION AND CONCLUSIONS}

Two kind of theories of the boundedly rational behavior are possible. Static theories focus on stationary behavior and do not include any explicit mechanism for temporal change. Similar to the rational choice theory, they embody something of a subject's cognitive analysis of the choice problem. Dynamic theories, on the other hand, explicitly model the fine-grain adjust- 
ments made by subjects to their recent experiences

Both types of theories originated in mathematical psychology. Static theories were originated by Luce (1959) and were based on the axiomatic approach to the characterization of the choice probabilities. Dynamic learning models where pioneered by Bush and Mosteller (1955). In these models learning is modelled as a Markov process on the choice set.

The main contribution of this paper is to argue that these two approaches are not always compatible. Namely, the axioms of the static approach are not supported by an explicit adjustment procedure. More precisely, for a broad class of locally improving processes a generic process does not satisfy IIA and a generic locally improving process that does satisfy IIA is not payoff monotone. Since, in my view, the dynamic approach is more fundamental the results of the paper imply that, when studying boundedly rational behavior, one should start with explicit formulation of the learning process. Since this contrasts with most current economic literature that utilizes probabilistic choice, it is interesting to study to what degree the results of this literature are robust to such a revision. 


\section{REFERENCES}

Alchian, A. A.: Uncertainty, evolution, and economic theory. Journal of Political Economy, LVIII, 211-221 (1950).

Anderson S. P., Goeree, J. K., Holt C. A.: Rent seeking with bounded rationality: An analysis of all-pay auction. Journal of Political Economy, 106, 828-853 (1998).

Anderson S. P., Goeree, J. K., Holt C. A.: Stochastic game theory: adjustment to equilibrium under bounded rationality. Working Paper, University of Virginia, (1999).

Anderson S. P., Goeree, J. K., Holt C. A.: Minimum-effort coordination games: stochastic potential and logit equilibrium. Games and Economic Behavior, 34, 177-199 (2001).

Arkes, H. R., Hammond, K. R.: Judgment and decision making: an interdisciplinary reader. Cambridge: Cambridge U. Press, 1986.

Arrow, K. J. and L. Hurwicz. : Stability of gradient process in n-person games, Journal of Society of Industrial and Applied Mathematics, 8, 280-94 (1960).

Basov, S.: Bounded rationality, reciprocity, and their economic consequences. 
Ph.D. Thesis, The Graduate School of Arts and Sciences, Boston University 2001.

Bush R.: and Mosteller, F.: Stochastic models for learning. New York: Wiley 1955.

Chen, H. C., Friedman, J. W., Thisse, J. F.: Boundedly rational Nash equilibrium: a probabilistic choice approach, Games and Economic Behavior, 18, $32-54(1997)$.

Conlisk, J.: Why bounded Rationality? Journal of Economic Literature, XXXIV, 669-700, (1996).

Estes, W. K.: Towards statistical theory of learning. Psychological Review, 5, 94-107 (1950).

Foster, D., Young, P.: Stochastic evolutionary game theory. Theoretical Population Biology, 38, 219-232 (1990).

Friedman, D., Yellin, J.: Evolving landscapes for population games, University of California Santa Cruz, mimeo (1997).

Friedman, D.: The evolutionary game model of financial markets, Quantitative Finance, 1, 177-185, (2000).

Fudenberg, D., Harris, C.: Evolutionary dynamics with aggregate shocks. Journal of Economic Theory, 57, 420-41 (1992). 
Gantmakher, F. R: The theory of matrices. New York : Chelsea, 1989.

Hogarth, R.: Judgment and choice: Psychology of decision, New York, Wiley 1980.

Ito, S.: Diffusion equation. American Mathematical Society, 1992.

Kagel, J. H., Roth, A. E.: Handbook of experimental economics. Princeton: Princeton University Press 1995.

Kahneman, D., Slovic P., Tversky A.: Judgment under uncertainty: heuristic and biases. Cambridge: Cambridge U. Press 1982.

Kandori, M., Mailath, G., Rob, R.: Learning, mutation and long run equilibria in games. Econometrica, 61, 29-56, (1993).

Luce, R. D.: Individual choice behavior, New York: Wiley 1959.

McKelvey, R. D., Palfrey, T. R.: Quantal response equilibria for normal form games. Games and Economic Behavior, 10, 6-38, (1995).

McKelvey, R. D., Palfrey, T. R.: Quantal response equilibria for extensive form games. Experimental Economics, 1, 9-41, (1998).

Nelson, R. R., Winter, S. G.: An evolutionary theory of economic change, Cambridge: Harvard University Press 1982.

Nisbett, R., Ross L.: Human inference: Strategies and shortcomings in the social judgment. Englewood Cliffs: Prentice-Hall 1980. 
Offerman, T., Schram, A., Sonnemans, J.: Quantal response models in steplevel public good games. European Journal of Political Economy, 14, 89-100, (1998).

Payne, J. W., Bettman, J. R., Johnson E. J.: Behavioral decision research: a constructive processing perspective. Annual Review of Psychology 43, 87$131(1992)$.

Pitz, G., Sachs N. J.: Judgment and decision: Theory and application. Annual Review of Psychology, 35,139-63 (1984).

Simon, H. A:. Administrative behavior; a study of decision-making processes in administrative organization. New York: Macmillan 1957.

Young, P.: The evolution of conventions, Econometrica, 61, 57-84, (1993). 